\title{
Documentos
}

\section{PAUTAS DE MANEJO CLÍNICO DE EMBARAZOS GEMELARES}

\author{
Pilar Valenzuela M. ${ }^{1}$, Jorge Becker V. ${ }^{1}$, Jorge Carvajal C. ${ }^{1}$ \\ 1 Unidad de Medicina Materno-Fetal, Departamento de Obstetricia y Ginecología, Facultad de Medicina, Pontificia \\ Universidad Católica de Chile.
}

\section{RESUMEN}

El embarazo gemelar conlleva un elevado riesgo de morbilidad y mortalidad perinatal. Teniendo el control prenatal una reconocida utilidad en disminuir la morbimortalidad perinatal, decidimos elaborar pautas de manejo clínico, basadas en evidencia, que orienten el seguimiento prenatal de embarazos gemelares. Realizamos una revisión de las publicaciones recientes, guías clínicas y textos clásicos de obstetricia obteniendo la información necesaria. Presentamos un listado de las recomendaciones y la calidad de evidencia que las sustenta, respecto de seis ítems: a. Reproducción asistida; b. Diagnóstico de embarazo gemelar; c. Manejo general del embarazo gemelar; $d$. Parto prematuro; e. Complicaciones específicas de embarazos gemelares; y f. Momento de interrupción y manejo del trabajo de parto.

\section{PALABRAS CLAVE: Embarazo múltiple, embarazo gemelar, guías clínicas}

\section{SUMMARY}

Multiple pregnancy has high risk of perinatal morbidity and mortality. Since antenatal care has been demonstrated as a useful tool in reducing perinatal risk, we decided to elaborate evidence based clinical guidelines, to facilitate antenatal care of women carrying multiple pregnancies. We reviewed recent publications, clinical guidelines and text books to obtain necessary information. Here we present a list of recommendations, and the quality of supporting evidence, about six items: a. Assisted reproduction; b. Diagnosis of multiple pregnancy; c. General care of multiple pregnancy; d. Preterm Labor; e. Specific complications of multiple pregnancy; $f$. Timing of delivery and labor management.

\section{KEY WORDS: Multiple pregnancy, twin pregnancy, guidelines}

\section{INTRODUCCIÓN}

La incidencia de embarazos múltiples ha aumentado dramáticamente en las últimas décadas en los países desarrollados, desde 1 por 100 nacidos vivos a 1 por 70 nacidos vivos, con un aumento de un $40 \%$ en la tasa de embarazos gemelares y un aumento de 3 a 4 veces en embarazos múltiples de alto orden. Este aumento en el número de embarazos múltiples es atribuible en mayor parte a la utilización de técnicas de reproducción asistida, pero también se explica por un aumento en la edad materna, ya que existe asociación entre la edad materna y la tasa de gestaciones múltiples concebidas espontáneamente (1).

El embarazo múltiple es un embarazo de alto riesgo fetal, pues tiene, entre otros problemas, mayor incidencia de prematurez, restricción del crecimiento fetal, malformaciones congénitas y muerte fetal in útero comparado con las gestaciones únicas. Existen además, complicaciones propias del embarazo gemelar como: crecimiento discordante, muerte in útero de alguno de los gemelos, síndrome de transfusión feto-fetal, gemelos monoamnióticos 
y perfusión arterial reversa en uno de los gemelos, que también dan cuenta de un mayor riesgo de estos embarazos. Por otra parte, se ha demostrado en embarazos gemelares que existe un mayor riesgo de morbimortalidad materna (2).

Siendo el control prenatal una herramienta de demostrada efectividad en disminuir el riesgo fetal, nos parece esencial contar con guías clínicas para el cuidado prenatal de embarazos gemelares, ofreciendo así un manejo estandarizado y basado en la evidencia. Estas guías clínicas están orientadas a lograr un diagnóstico oportuno del embarazo múltiple, situarlo en la categoría de riesgo correspondiente, pesquisar las posibles complicaciones oportunamente y ofrecer el mejor tratamiento disponible.

\section{MATERIAL Y MÉTODO}

Realizamos una búsqueda en las bases de datos: Cochrane Library, Tripdatabase y en el buscador Google Scholar. Combinamos las palabras claves "multiple pregnancy", "twin pregnancy" y "guidelines". Se excluyeron aquellas revisiones sistemáticas y guías clínicas que no eran específicas para embarazos gemelares y que se referían a intervenciones específicas en técnicas de reproducción asistida.

En Google Scholar accedimos al sitio de Geneva Foundation for Medical Education and Research donde encontramos las guías de manejo para embarazos gemelares de la Sociedad de Obstetras y Ginecólogos de Canadá y de la National Institute for Health and Clinical Excellence (NICE). En el Cochrane Library encontramos 34 revisiones sistemáticas de las cuales escogimos 7. En Tripdatabase encontramos 57 revisiones sistemáticas (de las cuales escogimos 6 que correspondían a las de Cochrane Library) y 52 guías clínicas de las cuales escogimos tres. Revisamos los capítulos correspondientes a embarazo múltiple de tres textos clásicos: Obstetricia de Williams (3), High Risk Pregnancy Management Options de James y cols (4), y Maternal Fetal Medicine de Creasy y Resnik (5).

De las revisiones sistemáticas, guías clínicas y textos clásicos obtuvimos referencias secundarias, al seleccionar aquellas publicaciones indicadas en las referencias, que impresionaron como fundamentales por su relevancia clínica o cita frecuente. Dividimos la información en 6 secciones:

I. Reproducción asistida

II. Diagnóstico de embarazo gemelar

III. Manejo general del embarazo gemelar

IV. Parto prematuro

V. Complicaciones específicas de embarazos gemelares
A. Gemelos discordantes por restricción del crecimiento fetal
B. Muerte fetal intrauterina
C. Anomalías congénitas
D. Síndrome de transfusión feto-fetal
E. Gemelos monoamnióticos
F. Secuencia de perfusión arterial reversa

VI. Momento de interrupción y manejo intraparto.

Para presentar la calidad de la evidencia disponible y clasificar las recomendaciones nos basamos en la clasificación de recomendaciones publicadas por el grupo "Canadian Task Force on the Periodic Health Exam" (Tabla I) (6); el resumen de las recomendaciones que estimamos importantes, se entrega en las Tablas II-VII, correspondientes a las diferentes secciones.

\section{RESULTADOS}

\section{REPRODUCCIÓN ASISTIDA}

Está bien establecido que la incidencia de gemelos y embarazos de alto orden es mayor, luego de reproducción asistida que de concepción y embarazo espontáneo. En la población general es difícil determinar la contribución exacta de las técnicas de reproducción asistida en la tasa de embarazos múltiples, ya que el registro de esta información no es riguroso. Para pacientes sometidas a técnicas de reproducción asistida, el riesgo del embarazo múltiple puede ser reducido con el uso conservador de la estimulación ovárica y con monitorización cautelosa, según las guías clínicas publicadas (7). Un consejo preconcepcional adecuado en relación a los riesgos del embarazo múltiple debiera darse a toda mujer en tratamiento de infertilidad (Tabla II).

\section{DIAGNÓSTICO DE EMBARAZO GEMELAR}

Existe clara evidencia de que el diagnóstico de embarazo gemelar mejora con el uso rutinario de la ultrasonografía. Lo mismo ocurre con el diagnóstico de corionicidad, estimándose que en el primer o segundo trimestre (< 16 semanas) la ultrasonografía determinará la corionicidad en el $100 \%$ de los casos (8). El momento ideal para evaluar la corionicidad de un embarazo gemelar es entre las 11 y las 14 semanas (9).

Los gemelos monocigóticos tienen un mayor riesgo de sufrir complicaciones que los dicigóticos, siendo la corionicidad y no la cigocidad lo que determina el resultado perinatal, por lo que se entiende la necesidad de determinar acuciosamente la corionicidad al momento del diagnóstico. En el examen ultrasonográfico realizado entre las 11 y 14 semanas de gestación se debe evaluar además la 
Tabla I

CLASIFICACIÓN DE RECOMENDACIONES, ADAPTADO DE: CANADIAN TASK FORCE ON THE PERIODIC HEALTH EXAM (6)

\section{NIVELES DE EVIDENCIA Y GRADOS DE RECOMENDACIÓN}

Calidad de la evidencia

I Evidencia obtenida de al menos un estudio randomizado controlado

II-1 Evidencia obtenida de estudios controlados de buen diseño, no randomiza dos.

II-2 Evidencia obtenida de estudios de cohorte bien diseñados (prospectivos o retrospectivos) o estudios de caso y control, preferentemente de más de un centro o grupo de investigación.

II-3 Evidencia obtenida de comparaciones entre tiempos o lugares con sin la intervención. Resultados dramáticos en experimentos no controlados tambié $n$ pueden ser incluidos en este categoría

III Opinión de autores respetados, basada en experiencia clínica, estudios descriptivos o reportes de comités de expertos.
Clasificación de las recomendaciones

A Existe evidencia categórica para apoyar la intervención en la práctica clínica.

B Existe discreta evidencia para apoyar la intervención en la práctica clínica.

C Existe pobre evidencia en relación a la inclusión/exclusión de la intervención, pero se pueden hacer recomendaciones en otros ámbitos.

D Existe discreta evidencia para no apoyar la intervención en la práctica clínica.

E Existe evidencia categórica para apoyar la recomendación de que la intervención no sea considerada.
Tabla II

\section{CUADRO RESUMEN DE RECOMENDACIONES SOBRE REPRODUCCIÓN ASISTIDA}

\section{REPRODUCCIÓN ASISTIDA}

1. Uso conservador de la estimulación ovárica y monitorización cautelosa (A).

2. Información sobre riesgos del embarazo múltiple a pacientes en tratamiento $(\mathrm{C})$.

vitalidad, como también descartar la presencia de una malformación congénita mayor y medir la translucencia nucal para determinar el riesgo de aneuploidía. Algunos autores han sugerido que la medición de la translucencia nucal podría predecir el riesgo de síndrome de transfusión feto-fetal (STFF) en gemelos monocoriales (10), aunque otros indican que esta práctica carece de utilidad (11).
Los gemelos monocoriales tienen tasas de mortalidad perinatal que son entre 2 y 6 veces mayores que las tasas de mortalidad de los bicoriales $(12,13)$ La placenta de los embarazos monocoriales presenta casi siempre anastomosis vasculares, las cuales pueden causar cambios de volumen sanguíneo significativos entre los gemelos, llevando a complicaciones como STFF, perfusión arterial reversa (TRAP) y transfusión fetal aguda luego del óbito de uno de los gemelos. Estas anastomosis están prácticamente ausentes en las placentas de los gemelares bicoriales. Conocer la corionicidad de un embarazo gemelar resulta fundamental, ya que se ha demostrado que la única medida que mejora el pronóstico de los gemelos monocoriales, especialmente en referencia a STFF, TRAP y restricción de crecimiento fetal (RCF) selectivo, es el diagnóstico acertado de la corionicidad, para identificar oportunamente estas complicaciones (Tabla III). 
Tabla III

\section{CUADRO RESUMEN DE RECOMENDACIONES SOBRE DIAGNÓSTICO DE EMBARAZO GEMELAR}

\section{DIAGNÓSTICO DE EMBARAZO GEMELAR}

1. Efectuar ultrasononido a las $10-14$ semanas de gestación para evaluar:
a. Vitalidad.
b. Corionicidad.
C. Malformación congénita mayor.
d. Translucencia nucal (B).

2. Determinar la corionicidad al momento del diagnóstico (II-3, C).

3. Momento ideal para determinar la corionicidad 10-14 semanas (II-3 C).

\section{MANEJO GENERAL DEL EMBARAZO GEME- LAR}

Existe controversia respecto a quien debe efectuar el control prenatal en los embarazos múltiples. Los revisores de la Cochrane Library en 2006 analizaron el rol de los policlínicos "especializados" para el control prenatal de embarazos gemelares y lo compararon con el control prenatal estándar, concluyeron que para determinar si el control prenatal especializado mejora los resultados de salud materna e infantil se requieren estudios randomizados controlados con un diseño y poder adecuado (14). Sin embargo, existe consenso en relación al control prenatal de embarazos gemelares. Se recomienda que los hospitales organicen el cuidado pre y pos natal en policlínicos multidisciplinarios guiados por un especialista con experiencia en manejo de gestaciones múltiples. Estos policlínicos deben contar con pautas clínicas específicas, con criterios claros de derivación, para permitir la referencia a centros especializados en medicina fetal cuando sea apropiado (Tabla IV).

\section{PARTO PREMATURO}

El parto prematuro es el principal contribuyente de la mayor tasa de morbimortalidad que presentan los embarazos gemelares en comparación a los embarazos únicos. Disminuir la tasa de prematurez en embarazos gemelares es uno de los principales objetivos del cuidado prenatal en estos embarazos. En un estudio realizado en los Estados Unidos se observó que el $57,4 \%$ de los niños producto de embarazos gemelares nació antes de las 37 semanas versus el 10,4\% de las gestaciones únicas (15). Predicción de riesgo: Existe buena evidencia avalando que la detección del acortamiento cervical por examen digital o por medición ultrasonográfi$\mathrm{ca}$, es un buen predictor del riesgo de parto prematuro $(16,17,18)$. Existe también buena evidencia de que la presencia cervicovaginal de fibronectina fetal (FF) predice un mayor riesgo de parto prematuro $(19,20,21)$, sin embargo, no existen estudios intervencionales bien diseñados para indicar la incorporación del tamizaje rutinario de FF al manejo prenatal de embarazos gemelares.

Prevención del parto prematuro: Se han descrito diversas conductas orientadas a prevenir el parto prematuro, a continuación, analizaremos la evidencia disponible para las conductas más relevantes.

Por largo tiempo se recomendó el reposo en cama en el hogar o en el hospital para disminuir la tasa de prematurez, sin embargo, los estudios randomizados controlados y metanálisis han demostrado que el reposo en cama en el hospital no disminuye la tasa de parto prematuro o la mortalidad perinatal en los gemelares (22). Se ha observado que en embarazos gemelares no complicados esta conducta

\section{Tabla IV \\ CUADRO RESUMEN DE RECOMENDACIONES SOBRE MANEJO GENERAL DEL EMBARAZO GEMELAR}

\section{MANEJO GENERAL DEL EMBARAZO GEMELAR}

1. Control pre y post-natal en policlínicos multidisciplinarios, especializados para embarazo múltiple, guiados por un especialista $(C)$.

2. Disponer de pautas clínicas específicas, con criterios claros de derivación a centros de medicina fetal (C).

3. El médico a cargo del policlínico debe tener experiencia en ultrasonido y manejo de embarazos múltiples. 
podría aumentar la incidencia de parto prematuro extremo y el estrés psicosocial materno (23), por otro lado, en mujeres con embarazo gemelar y alto riesgo de parto prematuro (por modificaciones y acortamiento cervical) no hay evidencia de que el reposo en cama disminuya el riesgo de parto prematuro (24). Por lo señalado anteriormente la hospitalización para reposo en cama como conducta rutinaria no es recomendable. Tampoco existe evidencia que apoye la restricción de actividades o abandono del trabajo.

No se ha demostrado en estudios observacionales y controlados que el cerclaje profiláctico de rutina disminuya el parto prematuro, más bien existe evidencia moderada en contra (25). Finalmente, el uso de tocolisis profiláctica con betamiméticos fue analizado en una revisión sistemática publicada en la Cochrane Library en 2008, los autores concluyeron que no hay evidencia suficiente para apoyar o desaconsejar su uso como una estrategia de prevención del parto prematuro en gemelares (26).

No existe consenso, respecto al uso de progestinas para prevenir el parto prematuro en embarazos gemelares. En 2007 un estudio randomizado controlado comparó la administración de 17 alfa hidroxiprogesterona intramuscular contra placebo y concluyó que no disminuía la tasas de partos prematuros y muerte fetal en pacientes con embarazos gemelares. Siendo éste estudio el de mejor diseño y con mayor número de pacientes disponibles, es que no se recomienda la administración de progestinas de forma rutinaria (27).

En cuanto al uso de corticoides ante la posibilidad inminente de parto prematuro, los grandes estudios que han demostrado la utilidad de su uso en disminuir las complicaciones perinatales en recién nacidos prematuros, se realizaron en gestaciones únicas, no existiendo estudios que hayan demostrado la utilidad en gestaciones múltiples, sin embargo, no existe una razón biológica por lo cual estos fármacos no beneficiarían a fetos en gestaciones múltiples, de modo que las pautas de administración de corticoides en gemelares, siguen las mismas líneas que las de embarazos únicos (Tabla V).

\section{COMPLICACIONES ESPECÍFICAS DE EMBA- RAZOS GEMELARES}

A. Restricción del crecimiento fetal selectivo. Los gemelares tienen una incidencia mayor de restricción de crecimiento fetal (RCF), por lo que debe ser cuidadosamente evaluado. El porcentaje de recién nacidos pequeños para la edad gestacional es de $27 \%$ en gemelos y $46 \%$ en triples (28). Aunque algunos autores consideran esta restricción como fisiológica, los gemelos pequeños para la edad gestacional al nacer tienen el mismo riesgo perinatal que los niños de embarazos únicos con esta condición (29). Por ello en la práctica clínica recomendamos usar tablas de crecimiento para embarazos únicos. En todo caso, los patrones de crecimiento fetal son más importantes que las mediciones absolutas. Ambos deben ser interpretados a la luz de la historia clínica, junto con los factores genéticos y ambientales que pueden afectar el crecimiento fetal.

\section{Tabla V \\ CUADRO RESUMEN DE RECOMENDACIONES SOBRE PARTO PREMATURO}

\section{PARTO PREMATURO}

1. Para predicción de riesgo es útil la detección de: modificaciones cervicales por examen digital (II-2, A), acortamiento cervical ultrasonográfico (II-1, A) y fibronectina fetal cervicovaginal (C). No se ha demostrado la utilidad de su uso rutinario.

2. Hospitalización para reposo en cama no es recomendable (I, E).

3. Restricción de actividades o abandono del trabajo no es recomendable (III, C).

4. Cerclaje profiláctico no recomendable (I, II-2, D).

5. Tocolíticos profilácticos no recomendable (I, II-2, D).

6. Progestinas profilácticas no recomendable (I, D).

7. Uso de corticoides según pautas existentes para gestaciones únicas (IV Grado B). 
La RCF selectiva ocurre en un $12 \%$ de los gemelos y se origina en una insuficiencia placentaria de mayor proporción, o exclusiva, para uno de los gemelos (30). El diagnóstico diferencial debe hacerse con el síndrome de transfusión feto-fetal o con aneuploidía, malformaciones congénitas o infecciones que afecten a uno de los gemelos. La RCF selectiva verdadera, es decir secundaria a insuficiencia placentaria, se traduce en un aumento de la morbimortalidad para el gemelo más pequeño, por lo cual es importante su diagnóstico oportuno y exacto.

Se define RCF selectivo como una diferencia de peso entre ambos fetos, mayor al $25 \%$ del peso del gemelo más grande, en presencia de un feto pequeño menor al percentil 10 o algún otro criterio para RCF (31). La discordancia de crecimiento se calcula dividiendo la diferencia de peso entre el gemelo más grande y el más pequeño por el peso del gemelo más grande y multiplicándolo por 100 . Se ha propuesto que el parámetro más exacto para detectar discordancia de crecimiento entre los gemelos es comparar las circunferencias abdominales: una diferencia mayor de $20 \mathrm{~mm}$ después de las 24 semanas tiene un valor predictivo positivo (VPP) de una discordancia al nacer de $83 \%$ (32).

El manejo de gemelos discordantes varía según la corionicidad, sin embargo, se recomienda la exclusión de aneuploidía e infecciones congénitas en todos los gemelos, independiente de la corionicidad. El estudio de líquido amniótico es útil para estudiar el cariotipo fetal e investigar infecciones según la sospecha clínica. Una ultrasonografía para evaluación detallada de la anatomía fetal permitirá descartar malformaciones mayores.

En gemelos bicoriales que presentan crecimiento discordante severo de instalación precoz es preferible no intervenir para maximizar las posibilidades del gemelo con crecimiento adecuado a expensas de la muerte espontánea del gemelo con RCF (33). La vigilancia fetal se realiza habitualmente combinando evaluación del crecimiento, perfil biofísico y velocimetría Doppler. La frecuencia y tipo de vigilancia fetal dependerá del grado de compromiso del gemelo con RCF. Al igual que en gestaciones únicas, en gemelos bicoriales, la disfunción útero-placentaria se asocia a una secuencia de cambios en el perfil biofísico y Doppler que sugiere un deterioro progresivo del gemelo afectado. Se debe considerar la administración de corticoides y pruebas de madurez pulmonar fetal si se contempla una interrupción temprana (34).

En gemelos monocoriales se debe excluir, en primer lugar, el STFF ya que su pronóstico y tratamiento son muy diferentes. No se ha determinado cual es el mejor método para evaluar el bienestar fe- tal en gemelos monocoriales con RCF selectiva; la velocimetría Doppler de la arteria umbilical no tiene el mismo valor pronóstico en gemelos monocoriales que en gestaciones únicas o gemelos bicoriales. El registro basal no estresante (RBNE) y el perfil biofísico impresionan actualmente como los mejores métodos disponibles para evaluar el bienestar fetal, aunque el óbito de uno de los gemelos puede ocurrir inesperadamente, a pesar de un RBNE y perfil biofísico normales (35).

$\mathrm{Si}$ se presume de modo inminente la muerte espontánea de uno de los gemelos, previo a la viabilidad fetal, el feticidio selectivo por oclusión del cordón umbilical podría proteger mejor al gemelo con crecimiento adecuado de los efectos colaterales del óbito espontáneo. Esta conducta en Chile no es aceptable de acuerdo a la legalidad vigente.

Se ha realizado con éxito la coagulación con láser de las anastomosis vasculares (división placentaria) en embarazos gemelares monocoriales complicados con RCF selectiva y grave compromiso del feto (flujo diastólico de arteria umbilical ausente o reverso). Al comparar esta intervención con el manejo expectante, se observó una proporción mayor de muerte del gemelo con RCF y protección para el gemelo normal ante la muerte del gemelo con RCF (36). En general, frente a la confirmación de una RCF selectiva, se debe indicar la interrupción electiva a las 32 semanas, luego de la administración de corticoides o confirmación de madurez pulmonar.

En gemelos de corionicidad desconocida es posible recurrir a métodos adicionales para determinar la corionicidad como el examen de ADN y la búsqueda ultrasonográfica de anastomosis vasculares. El examen de ADN mediante amniocentesis para determinar la cigocidad permite descartar la monocorionicidad si los gemelos resultan genéticamente diferentes (37). La detección ultrasonográfica de anastomosis con su característica onda bidireccional permite confirmar la monocorionicidad con un $100 \%$ de confianza (38). Si no es posible determinar la corionicidad se debe manejar el embarazo como si fuera monocorial.

B. Muerte fetal intrauterina. Los fetos en embarazos gemelares tienen mayor probabilidad de fallecer in útero que los fetos en embarazos únicos (39). El diagnóstico de óbito de uno de los gemelos debiera hacerse cuando los remanentes fetales son claramente identificables o cuando un examen ultrasonográfico ulterior confirma el óbito o la desaparición de uno de los fetos previamente conocido. En embarazos bicoriales las mejores estimaciones de tasa de muerte de uno de los gemelos se obtienen del seguimiento de embarazos gemelares resultantes de IVF, y fluctúan entre un 10 y $20 \%$ según la edad materna, la mayoría ocurre antes de las 12 
semanas (40). En gemelos monocoriales, entre las 10 y 14 semanas, es más frecuente la muerte de ambos gemelos que la muerte de uno sólo de ellos (41). Desde las 14 semanas en adelante la muerte de uno de los gemelos ocurre en el $2 \%$ de los embarazos bicoriales y en un $4 \%$ de los monocoriales versus un $1,5 \%$ de mortalidad en el total de embarazos (42).

El pronóstico para el feto remanente depende fundamentalmente de la corionicidad. En gemelos bicoriales la muerte de uno de los gemelos en el primer trimestre tiene por lo general una evolución favorable; si ésta ocurre en el segundo o tercer trimestre, se asocia a muerte o discapacidad del gemelo sobreviviente en un 5 a $10 \%$ de los casos (42); en tal caso, el pronóstico dependerá de la edad gestacional al momento de la muerte fetal, con una mayor supervivencia del segundo gemelo al acercarse a las 37 semanas de gestación (43). En los monocoriales la muerte de uno de los gemelos resulta en la muerte de ambos en un 10 a $25 \%$ y en daño cerebral entre un 25 y $45 \%(44,45)$. El conocer la corionicidad es fundamental en el manejo de la muerte intrauterina de uno de los gemelos (46).

Existen recomendaciones de manejo que se aplican a todos los gemelos, independiente de la corionicidad. Respecto al cuidado del gemelo sobreviviente, se debe continuar con la vigilancia de bienestar fetal. Respecto al bienestar materno, se debe administrar gammaglobulina antiRh profiláctica si la paciente es Rh negativo. Si la edad gestacional es mayor a 20 semanas y la muerte data más de 4 semanas se debe evaluar el estado de coagulación materno, evaluando fibrinógeno y recuento plaquetario de modo seriado. Resulta prudente ofrecer apoyo psicológico a la madre y a su familia.

En gemelos monocoriales se debe considerar el uso de velocimetría Doppler en arteria cerebral media para detectar anemia, lo cual puede predecir el riesgo de daño cerebral. En aquellos casos de anemia severa del gemelo sobreviviente es posible realizar transfusión intrauterina, logrando una sobrevida de un 58\% (47). El gemelo sobreviviente debiera ser seguido con ultrasonografía, y si esta resulta normal, se recomienda la evaluación del cerebro fetal con resonancia nuclear magnética, 2 a 3 semanas después de la muerte del co-gemelo. Se debe informar a los padres de la morbilidad a largo plazo de esta condición. Los embarazos monocoriales complicados por la muerte de un gemelo deben ser referidos y evaluado en un centro de medicina fetal avanzada.

C. Anomalías congénitas. Las anomalías congéni- tas son más frecuentes en embarazos gemelares que en embarazos únicos, con tasas reportadas de hasta un 4,9\% (48). La presencia de anomalías fetales, es inicialmente mejor evaluada entre las 16 y 20 semanas. Lamentablemente la mayoría de los estudios no informa la incidencia según cigocidad, aunque pareciera que la incidencia de anomalías en gemelos dicigóticos es la misma que en fetos únicos, la tasa es 2 a 3 veces mayor en gemelos monocigóticos. En gemelos monocigóticos es rara (menor a $20 \%$ ) la concordancia de anomalías estructurales y son frecuentes los reportes de discordancia para enfermedades genéticas (49). La prevalencia global de anomalías cardiacas en gemelos monocoriales es alrededor de un $4 \%$ versus el $0,6 \%$ observado en la población general, en gemelos monocoriales con STFF es aún mayor, alcanzando un $7 \%(50)$.

En los gemelos dicigóticos el riesgo de síndrome de Down (relacionado a la edad materna) de cada gemelo es independiente y similar que para fetos únicos, por lo tanto el riesgo de tener al menos un niño con síndrome de Down es el doble que para un embarazo único. En embarazos gemelares monocoriales el riesgo es el mismo para ambos gemelos y similar al de fetos únicos, aunque se ha descrito discordancia en la expresión para la gran mayoría de las aneuploidías humanas. El feticidio selectivo, si un solo feto está afectado, puede causar la pérdida del gemelo no afectado. Por cierto, por motivos éticos y de respeto a la vida humana, no recomendamos ningún tipo de aborto o feticidio.

Como concepto general, los exámenes de tamizaje debieran tener una buena sensibilidad y una tasa aceptable de falsos positivos, ya que las pruebas invasivas tienen un mayor riesgo y son técnicamente más difíciles en embarazos múltiples. El tamizaje bioquímico materno para pesquisa de aneuploidía en gemelos tiene tasas de detección muy bajas; por otro lado indica el riesgo del embarazo global de tener al menos un feto afectado y no permite identificar específicamente cual de los fetos tiene la anomalía. Por lo recién señalado el tamizaje bioquímico no está recomendado (51). Por el contrario, la medición de la translucencia nucal en el primer trimestre tiene una buena sensibilidad e identifica al feto con riesgo aumentado, por lo cual es el examen de elección para determinar el riesgo de aneuploidía (52). Si existe un riesgo elevado de aneuploidía y se quiere determinar el cariotipo fetal mediante procedimientos invasivos como amniocentesis o biopsia de vellosidades coriales, se debe tener en cuenta que las tasas de pérdida fetal con éstas pruebas invasivas no es conocida en gemelos. Si se realiza un procedimiento invasivo resulta 
de vital importancia conocer con certeza de cual feto es la muestra obtenida. Las pruebas invasivas deben ser realizadas en gemelos de acuerdo al estándar usual de manejo de gestaciones únicas.

De modo específico, en los gemelos monocoriales, por presentar una mayor tasa de malformaciones cardíacas, se estima indispensable efectuar una ultrasonografía con visión detallada del corazón fetal.

Los gemelos unidos o siameses corresponden a una anomalía congénita exclusiva de embarazos gemelares y afecta a uno de 200 embarazos monocigóticos (53). Por lo general el diagnóstico de esta anomalía es prenatal (54) y su manejo dependerá de un equipo multidisciplinario. El pronóstico de los gemelos siameses depende principalmente del sitio de unión y del tipo de órganos compartidos (53). El manejo de esta condición se adecua caso a caso, de modo que escapa a las posibilidades de este artículo entregar recomendaciones generales.

$D$. Síndrome de transfusión feto-fetal (STFF). Este es una complicación exclusiva de los embarazos múltiples monocoriales. En la mayoría de los embarazos monocoriales existe una transfusión equilibrada entre los fetos a través de las anastomosis. Entre un 10 y $15 \%$ de los embarazos monocoriales presentan un desbalance crónico en el flujo neto, resultando en STFF. En el gemelo donante se desarrolla hipovolemia, oliguria y oligoamnios (OHA) produciendo el fenómeno de feto suspendido (stuck twin). En el gemelo receptor se produce hipervolemia, poliuria y polihidroamnios (PHA), desarrollando sobrecarga circulatoria e hidrops. EI STFF se presenta generalmente entre las 15 y 26 semanas, su diagnóstico es ultrasonográfico, aceptándose hoy en día los siguientes criterios diagnósticos (55):

1. Diagnóstico acertado de corionicidad antes de las 16 semanas.

2. Diagnóstico de la enfermedad entre las 16 y 26 semanas de embarazo (sobre esa edad gestacional, también puede iniciarse pero su evolución es más benigna y el soporte neonatal mejora el pronóstico).

3. Feto receptor con polihidroamnios, definido como la presencia de un bolsillo de líquido amniótico mayor de $8 \mathrm{~cm}$ entre las 16 y 20 semanas o de $10 \mathrm{~cm}$ entre las 21 y las 26 semanas, sumado a la presencia de una imagen de vejiga muy distendida.

4. Feto donante con oligohidroamnios, definido como la presencia de un bolsillo de líquido amniótico inferior a $2 \mathrm{~cm}$, acompañado por la ausencia de imagen vesical (vejiga colapsada) durante la mayor parte de la exploración.

El Doppler fetal es una herramienta útil, ya que permite evaluar el estado hemodinámico de los gemelos, sin embargo, sus alteraciones no están incluidas dentro del diagnóstico de STFF, ya que esta enfermedad se puede presentar con Doppler fetal normal en su inicio.

Los gemelos que presentan STFF severo en el segundo trimestre pueden tener desbalance hemodinámico desde el primer trimestre. Para pesquisar la enfermedad en etapas tempranas se debe realizar, a todos los gemelos monocoriales, ultrasonografía seriada, cada 2 semanas, desde las 16 semanas y hasta las 26 semanas de gestación. Ante la pesquisa de signos sugerentes de STFF se debe derivar a la paciente a un centro terciario que cuente con un obstetra especialista en medicina fetal.

Para categorizar la severidad de la enfermedad se utiliza el sistema de Quintero y cols (56), que clasifica el STFF en 5 etapas. Las etapas de I a $\mathrm{V}$ se basan en un empeoramiento progresivo de las características clínicas encontradas en la ultrasonografía. Sin tratamiento el STFF tiene una mortalidad cercana al $100 \%$. El PHA se asocia a aborto espontáneo y parto prematuro extremo, la muerte de los gemelos puede ocurrir secundaria a falla cardiaca del receptor o perfusión disminuida del donante. Ambos gemelos presentan un elevado riesgo (alrededor de un 35\%) de lesiones de la sustancia blanca cerebral (57). Según el tratamiento efectuado la frecuencia de parálisis cerebral y retraso del desarrollo global varían entre un 4 y 26 $\%$ (58).

Las alternativas terapéuticas para el STFF incluyen: manejo expectante y terapias intervencionales. El manejo expectante tiene muy mala tasa de supervivencia, por lo cual existe consenso en que se debiera ofrecer tratamiento. Las alternativas terapéuticas utilizadas con mayor frecuencia son: amniodrenaje seriado, fotocoagulación con láser de las anastomosis placentarias y feticidio selectivo. Se ha utilizado también la septostomía como medida terapeútica, no obstante, hoy en día este procedimiento se encuentra obsoleto y no es una alternativa de tratamiento.

El amniodrenaje seriado permite controlar el volumen de líquido amniótico, no requiere una gran habilidad técnica y está ampliamente disponible. El amniodrenaje prolonga el embarazo y mejora la condición fetal al disminuir la presión intrauterina, aunque no protege al gemelo sobreviviente en caso de muerte de uno de los gemelos y es solo efectivo en casos leves de STFF. En una revisión que incluyó a 223 pares de gemelos tratados con amniodrenaje la tasa global de sobrevida fue de $78 \%$ (sobrevida de ambos gemelos $48,4 \%$, sobrevida de al menos un gemelo $70,8 \%$ ), en esta revisión se encontró una prevalencia de alteraciones en la 
evaluación intracraneana por imágenes en un $24 \%$ de los receptores y en un $25 \%$ de los donantes sobrevivientes (59).

La coagulación con láser de los vasos anastomóticos está dirigida a tratar el origen de la enfermedad, pues la oclusión de los vasos comunicantes permitiría detener el fenómeno de transfusión feto-fetal. El estudio randomizado controlado Eurofetus (60), comparó la amnioreducción seriada con la coagulación con láser en STFF en embarazos menores de 26 semanas. En este estudio la sobrevida, edad gestacional al parto y peso al nacer fueron significativamente mayores y la frecuencia de leucomalasia periventricular significativamente menor en el grupo tratado con láser comparado con amniodrenaje. En una revisión de las distintas series publicadas, que incluyó sobre 1300 casos de STFF, se observó una sobrevida global aproximada de $65 \%$, siendo de $50-70 \%$ para ambos fetos, de $70-93 \%$ para al menos uno y mortalidad de ambos en un $15-20 \%(61)$.

Respecto a las alternativas de tratamiento en STFF, los autores de la revisión sistemática para la Cochrane Library en 2007, concluyen que la coagulación endoscópica con láser de vasos anastomóticos, debiera ser considerada para mejorar los resultados perinatales en todas las etapas del síndrome de transfusión feto-fetal. Los autores hacen hincapié en la necesidad de contar con más estudios para evaluar el efecto del tratamiento en etapas más leves de la enfermedad (Quintero I y II) y conocer los resultados a largo plazo de los estudios evaluados para la revisión (62).

E. Gemelos monoamnióticos. Los gemelos monocoriales monoamnióticos resultan cuando la división de la masa celular interna ocurre después del noveno día de la fecundación. Los gemelos monocoriales son muy poco frecuentes, ocurren en 1 de cada 10.000 embarazos y en un $5 \%$ de los gemelos monocoriales (63). Dentro de los gemelos monoamnióticos se incluyen los gemelos unidos o siameses con una incidencia de 1 en 50.000 embarazos.

El diagnóstico de gemelos monoamnióticos se efectúa con ultrasonografía en el primer trimestre al observar 2 fetos y una sola cavidad amniótica. El entrecruzamiento de los cordones es patognomónico de gemelos monoamnióticos y es evaluable desde el primer trimestre con el uso de Doppler pulsado en el ovillo de vasos, demostrando la presencia de 2 frecuencias cardiacas distintas (64). Otras características ultrasonográficas que apoyan el diagnóstico son:

a) ausencia de membrana amniótica divisoria

b) presencia de placenta única

c) ambos fetos del mismo sexo d) cantidad adecuada de líquido amniótico alrededor de cada feto

e) ambos fetos se mueven libremente dentro de la cavidad uterina

Los gemelos monoamnióticos tienen una alta tasa de mortalidad (hasta un 50\%) que se atribuye al entrecruzamiento de cordones, nudos de cordón, anomalías congénitas y prematurez. Los gemelos monoamnióticos tienen un alto riesgo de malformaciones congénitas, alcanzando una frecuencia de 38 a 50\%, afectando habitualmente a un solo gemelo.

La muerte fetal intrauterina es el principal componente de la mortalidad perinatal en gemelos monoamnióticos sin malformaciones, siendo frecuente la muerte de ambos gemelos. El riesgo basal de muerte intrauterina en gemelos monoamnióticos alcanza hasta un $70 \%$ (65) y disminuye a un $2 \%$ con vigilancia antenatal estricta (66). Las causas más frecuentes de muerte fetal intrauterina son: atrapamiento de cordón umbilical y síndrome de transfusión feto-fetal aguda con desbalance hemodinámico.

Los gemelos monoamnióticos requieren una estrecha vigilancia antenatal y deben ser manejados en un centro terciario que cuente con un obstetra especialista en medicina fetal. Dada la alta frecuencia de malformaciones congénitas en este grupo de gemelos se debe realizar ultrasonografía acuciosa para evaluar la anatomía fetal a las 16-18 semanas. El plan de manejo antenatal debe incluir monitorización del bienestar fetal con RBNE y PBF al menos 1 vez por semana desde las 26 semanas (67). Esta alta frecuencia de monitorización es justificada porque los eventos que causan la muerte de los gemelos tienden a ser repentinos, por lo que la muerte fetal no es evitable. La alteración de las ondas de flujo Doppler medidas en la arteria umbilical podría predecir compresión del cordón umbilical, pero, al igual que para gemelos monocoriales los valores predictivos de estas alteraciones son distintos que para gestaciones únicas, por lo cual su uso rutinario no está recomendado (68). No se ha dilucidado si éstas pacientes se benefician de la hospitalización rutinaria, ésta decisión debiera ser individualizada para cada paciente según su evolución (69).

Se ha descrito en la literatura que el momento ideal para la interrupción es a las 32 semanas de edad gestacional, posteriormente la mortalidad fetal aumenta y se produce una marcada disminución de las complicaciones neonatales (70). Se recomienda la interrupción por operación cesárea electiva ya que se evitaría el atrapamiento de cordón o el corte inadvertido del cordón del segundo gemelo que podría estar alrededor del cuello del primero. Lógicamente, 
se debe administrar corticoides con el fin de disminuir las complicaciones neonatales.

F. Secuencia de perfusión arterial reversa. La secuencia de perfusión arterial reversa en gemelos (TRAP), también conocida como gemelo acárdico es una patología exclusiva a los gemelos monocoriales, afectando al $1 \%$ de ellos. La sangre fluye desde la arteria umbilical de un gemelo (gemelo bomba) en sentido reverso hacia la arteria umbilical del otro gemelo (gemelo acárdico). Esta patología se origina por la presencia de anastomosis arterio-arteriales y produce hipoxemia del gemelo perfundido (gemelo acárdico); la hipoxemia crónica produce grados variables de deficiencia en el desarrollo de la cabeza, corazón y extremidades superiores del gemelo acárdico. Existe un desarrollo al menos parcial del abdomen y extremidades inferiores, que son perfundidos por el gemelo bomba, que tiene el riesgo de presentar insuficiencia cardiaca congestiva y parto prematuro extremo secundario a polihidroamnios. El diagnóstico se realiza con certeza utilizando ultrasonografía en el primer trimestre (71). La secuencia TRAP se caracteriza por encontrarse, en un embarazo gemelar monocorial, uno de los fetos con múltiples anomalías, que presenta crecimiento y movimiento pero sin función cardiaca propia. La presencia de movimiento cardíaco no excluye el diagnóstico ya que el gemelo acárdico puede tener un corazón rudimentario o presentar pulsaciones transmitidas del corazón del gemelo bomba.

Dado que se trata de una patología muy infrecuente, es que la historia natural de la enfermedad no se conoce con exactitud. En una revisión de la literatura que incluyó 216 casos manejados conservadoramente se observó que sin tratamiento, la sobrevida global del gemelo bomba es de un $60 \%$. La muerte intrauterina ocurre en un $25 \%$, la presencia de $\mathrm{PHA}$ en un $50 \%$ y el parto prematuro en un $80 \%$ (72). En algunos casos ocurre resolución espontánea de la secuencia TRAP al cesar completamente el flujo hacia el gemelo acárdico. Es posible predecir, en cierta medida, el resultado perinatal de la secuencia TRAP: un tamaño proporcionalmente pequeño del gemelo acárdico respecto del gemelo bomba y grandes diferencias en el flujo de las arterias umbilicales, medido con velocimetría Doppler (sugiriendo escaso flujo hacia el gemelo acárdico), otorgan un mejor pronóstico (73). Se ha propuesto una clasificación ultrasonográfica que agrupa a los gemelos complicados por secuencia TRAP (74), esta clasificación considera el tamaño del gemelo acárdico y el impacto cardiovascular en el gemelo bomba. Según la clasificación, se propone el mo- mento y tipo de intervención a efectuar (74).

La modalidad de tratamiento de embarazos gemelares complicados por la secuencia TRAP es controversial. El objetivo del tratamiento es aumentar la probabilidad de sobrevida y eventualmente lograr un parto a término del gemelo bomba. Se recomienda tratamiento intervencional ante la presencia de signos de mal pronóstico (gran tamaño del gemelo acárdico y alto flujo hacia el gemelo acárdico), si no ha ocurrido interrupción espontánea del flujo hacia el feto acárdico a las 16 semanas. Las distintas alternativas de terapia invasiva tienen precisamente como objetivo interrumpir el flujo hacia el gemelo acárdico. Para ello, existen procedimientos de oclusión del cordón y procedimientos de oclusión vascular intra-fetal. Es posible ocluir el cordón del gemelo acárdico mediante procedimientos guiados por ultrasonografía o por visión endoscópica directa del feto (fetoscopia). Con guía ultrasonográfica es posible introducir distintos materiales en los vasos del cordón o coagularlo con electrodo monopolar o bipolar. Mediante fetoscopia es posible efectuar la ligadura directa del cordón. Si la oclusión del cordón es técnicamente muy difícil, entonces es necesario realizar procedimientos de oclusión vascular intra-fetal, dirigidos a la aorta abdominal o los vasos pelvianos del gemelo acárdico. La oclusión vascular intra-fetal se ha realizado inyectando sustancias esclerosantes, mediante coagulación con electrodo monopolar o láser guiado por ultrasonido y mediante ablación por radiofrecuencia (74).

No existen estudios randomizados controlados que comparen los resultados perinatales luego de intervención versus ausencia de intervención. La evidencia disponible proviene de estudios pequeños con escaso número de pacientes (75-79). Al analizar estos estudios se observa que sin intervención la sobrevida del gemelo bomba es de un $60 \%$ versus un $75 \%$ con intervención. No se ha demostrado diferencia entre los procedimientos de oclusión de cordón y los procedimientos de oclusión vascular intra-fetal, aunque existe una tendencia a mayor sobrevida con las técnicas intra-fetales. Siendo estas últimas técnicamente más sencillas y más ampliamente disponibles, debieran ser la alternativa de elección si se opta por una terapia invasiva.

Ya sea en los casos de manejo expectante (interrupción espontánea del flujo hacia el gemelo acárdico) o en aquellos intervenidos, si no existe alteración en el bienestar fetal, el embarazo puede continuar hasta el término. La vía de parto, en estos casos, dependerá de factores habituales en el manejo obstétrico (Tabla VI). 
Tabla VI a

CUADRO RESUMEN DE RECOMENDACIONES SOBRE COMPLICACIONES ESPECÍFICAS DE EMBARAZOS GEMELARES

RESTRICCIÓN DEL CRECIMIENTO FETAL SELECTIVA

Todos los gemelos:

1. Excluir aneuploidía, anomalías estructurales e infecciones congénitas.

Gemelos bicoriales:

1. En RCF selectiva severa de instalación precoz es preferible no intervenir para maximizar las posibilidades del gemelo con crecimiento adecuado a expensas de la muerte espontánea del gemelo con RCF (III, B).

2. Vigilancia fetal mediante evaluación del crecimiento, PBF y velocimetría Doppler.

3. Vigilancia y momento de intervención según compromiso del gemelo con RCF.

4. Administración de corticoides y las pruebas de madurez pulmonar fetal si se contempla una interrupción temprana (III,B).

Gemelos monocoriales:

1. Recordar que las anomalías, enfermedades genéticas y grado de afectación secundaria a infección pueden no ser concordantes en gemelos monocoriales (II-1, B).

2. Excluir síndrome de transfusión feto-fetal (II-1, B).

3. Considerar derivación a centro terciario.

4. Coagulación endoscópica con láser de las anastomosis vasculares en aquellos casos en que el gemelo pequeño se encuentre muy comprometido.

5. Interrupción a las 32 semanas, con corticoides o confirmación de madurez pulmonar.

6. Evaluación del bienestar fetal con registro basal no estresante (RBNE) y PBF (III, B).

Gemelos de corionicidad desconocida:

1. Examen de ADN para excluir la monocorionicidad (III, B).

2. Búsqueda de anastomosis arterio-arteriales para confirmar monocorionicidad (III, B).

3. Si la corionicidad no se logra confirma, manejo como gemelos monocoriales.

\section{MUERTE FETAL INTRAUTERINA}

Todos los gemelos:

1. Buscar signos de amenaza de aborto y parto prematuro extremo (III, B).

2. Vigilancia fetal en gemelo sobreviviente (III, B).

3. Corticoides si se contempla la posibilidad de parto prematuro (I, A).

4. Rhogam profiláctico si madre es Rh negativo.

5. Medición de fibrinógeno y recuento plaquetario si la edad gestacional es mayor a 20 semanas y la muerte data más de 4 semanas (IV, C)

6. Consejo y apoyo psicológico a lapaciente y a su familia (III, B).

Gemelos monocoriales:

1. Doppler de arteria cerebral media para detectar anemia, y predecir el riesgo de daño cerebral (III, B):

2. Seguimiento del sobreviviente con ultrasonografía, y si resulta normal, evaluación del cerebro fetal con RNM 2-3 semanas después de la muerte del co-gemelo (III, B).

3. Derivación a un centro de medicina fetal (B). 


\section{Tabla VI b}

\section{CUADRO RESUMEN DE RECOMENDACIONES SOBRE COMPLICACIONES ESPECÍFICAS DE EMBARAZOS GEMELARES}

\section{ANOMALÍAS CONGÉNITAS}

1. Tamizaje bioquímico para pesquisa de aneuploidia no recomendado.

2. Translucencia nucal método de elección para detectar aneuploidías (B).

3. Amniocentesis o biopsia de vellosidades coriales uso restringido (II-3, C).

4. Incluir ecocardiografía en monocoriales (B).

\section{SÍNDROME DE TRANSFUSIÓN FETO-FETAL}

1. Monocoriales evaluados con ultrasonografía por un especialista en medicina fetal para evaluar el crecimiento y/o aparición de signos sugerentes de STFF (III, C).

2. Evaluación ultrasonográfica cada 2 semanas entra las 16 y las 26 semanas.

3. Usar criterios diagnósticos específicos.

4. Derivación urgente a un centro especializado (II-2, B).

5. Amniodrenaje seriado es alternativa de tratamiento en casos leves (II-2, B).

6. La coagulación de anastomosis con láser mediante fetoscopía es el tratamiento de primera línea (I-2, A), especialmente antes de las 26 semanas (A).

\section{GEMELOS MONOAMNIOTICOS}

1. El entrecruzamiento de los cordones, es patognomónico de monoaminióticos (III, B).

2. Ultrasonografía detallada de la anatomía fetal para excluir malformaciones congénitas.

3. Derivación a un centro especializado.

4. Vigilancia antenatal desde las 26 semanas mediante RBNE y PBF desde una vez al día hasta una vez por semana (III, B).

5. Interrupción a las 32 semanas con corticoides o madurez pulmonar (II-1, B).

6. Interrupción por operación cesárea electiva (II,B).

\section{SECUENCIA TRAP}

1. Diagnóstico ultrasonográfico desde el primer trimestre.

2. Intervención ante la presencia de signos de mal pronóstico si no ha ocurrido interrupción espontánea del flujo hacia el gemelo acárdico (<16 sem) (II,B).

3. Oclusión vascular intrafetal es el procedimiento de elección.

4. Parto a término si se mantiene el estado de bienestar fetal.

5. La vía de parto depende de factores obstétricos (III,B).

6. Derivación a centro de referencia ante la sospecha o confirmación. 


\section{MOMENTO DE INTERRUPCIÓN Y MANEJO INTRAPARTO}

A. Momento de interrupción. Para determinar el momento óptimo de interrupción de un embarazo gemelar se debe tomar en cuenta la edad gestacional y peso estimado. Al analizar la mortalidad fetal por peso de nacimiento y edad gestacional, para embarazos gemelares, se observó que el menor riesgo de muerte fetal se encuentra entre los 2500 - 2800 gramos y entre las 36 - 37 semanas (80). Datos poblacionales muestran un aumento de la morbimortalidad perinatal que comienza a las 3839 semanas, por lo que resultaría prudente considerar la interrupción del embarazo antes de esta edad gestacional (81).

Los revisores de la Cochrane Library analizaron la evidencia para sustentar la interrupción de los embarazos gemelares desde las 37 semanas de gestación y concluyeron que el estudio disponible es demasiado pequeño para detectar las mediciones de resultados de interés, consecuentemente hay datos insuficientes para apoyar la práctica de interrupción electiva desde las 37 semanas en adelante en un embarazo gemelar sin complicaciones $(82,83)$. Sin embargo, en la práctica clínica habitual se tiende a interrumpir electivamente los embarazos gemelares no complicados entre las 37 y 38 semanas. Los embarazos gemelares complicados (por patología materna o fetal) se interrumpirán en el momento apropiado, buscando el balance entre los riesgos de la prematurez y el riesgo de daño fetal. De ser posible, se intentará esperar hasta las 36-37 semanas de edad gestacional. Como se indicó, los embarazos gemelares monoamnióticos debieran interrumpirse a las 32 semanas.

B. Manejo intraparto. Es importante analizar la evidencia existente respecto al cuidado intraparto de embarazos gemelares, ya que la frecuencia de estos va en aumento y la presencia de dos fetos amplía el número de alternativas de manejo del parto.

Las complicaciones del trabajo de parto y parto son más frecuentes en embarazos gemelares que en embarazos únicos. Existe una mayor frecuencia de prematurez y emergencias obstétricas, por lo cual es perentoria la cercanía o incluso presencia en el hospital de un médico obstetra, anestesiólogo, pediatra o neonatólogo, enfermeras matronas e instrumentista quirúrgico.

El parto vaginal de gemelos debe atenderse en un centro que disponga de monitorización intraparto continua y acceso inmediato a operación cesárea. La monitorización electrónica de frecuencia cardiaca fetal de ambos gemelos debe asegurar que ambos gemelos están siendo monitorizados individualmente. El diagnóstico de posición y presentación de cada feto al ingresar la paciente en trabajo de parto se debe confirmar por ultrasonido. Si la paciente no tiene una ultrasonografía reciente (2 a 3 semanas), es necesario realizarla al ingreso para estimar el peso fetal y facilitar la toma de decisiones, por ejemplo, en una paciente con presentación cefálica-no cefálica para decidir la vía de parto del segundo gemelo. La ultrasonografía también será de utilidad en la sala de partos, para evaluar la frecuencia cardíaca y eventual cambio de presentación del segundo gemelo luego del parto del primero.

En una revisión con cerca de 16.000 gemelos la tasa de cesárea fue de $47 \%$ (84). Las indicaciones de operación cesárea electiva en embarazos gemelares son las mismas que para gestaciones únicas, pero proporcionalmente son diferentes. La primera causa de indicación de cesárea en gemelos es la distocia de presentación (47\%). Existe consenso, en el caso particular de los gemelos monoamnióticos y gemelos unidos o siameses, en que la interrupción debe ser por cesárea. Es probable que la tasa de cesáreas en gemelos continúe aumentando, debido a que han disminuido las complicaciones asociadas a cesáreas electivas, la falta de experiencia de los médicos en atención de partos en podálica y la falta de estudios randomizados controlados que evalúen parto vaginal versus cesárea para el segundo gemelo distócico. Se postula además el beneficio de la cesárea versus el parto normal, pues se ha demostrado mayor morbilidad del segundo gemelo, y se cree que esta diferencia puede ser corregida por la cesárea. En embarazos de término cuando ocurre un parto distinto a cesárea electiva, el riesgo absoluto de mortalidad relacionada al parto para el segundo gemelo es de 1 en 270 , cifra muy superior a la observada para el primer gemelo y para embarazos únicos (85).

El manejo intraparto dependerá fundamentalmente de la presentación fetal, siendo tres las distintas combinaciones posibles: a) cefálica/cefálica (45\%); b) cefálica/no-cefálica (34\%); c) no-cefálica/ otra $(21 \%)(84)$.

Cefálica-cefálica: Es la presentación más frecuente y habitualmente posible obtener un parto vaginal para ambos fetos. La mayor serie reportada mostró un $73 \%$ de parto vaginal para ambo fetos, con un $19 \%$ de tasa de cesárea para ambos fetos (84). En menos de un $10 \%$ de los partos ocurre algún evento luego del parto del primer gemelo. En este reporte se requirió de operación cesárea para el segundo gemelo en un $6 \%$ de los casos. Las indicaciones de cesárea para el segundo gemelo incluyen prolapso de cordón, sufrimiento fetal, desprendimiento 
prematuro de placenta normoinserta y distocia de presentación. Los médicos deben estar preparados para realizar una operación cesárea de urgencia o considerar la extracción en podálica si la paciente cumple los requisitos.

Existe consenso sobre el tiempo a esperar para el parto del segundo gemelo, éste no debiera sobrepasar los 30 minutos. Esta recomendación se basa en la demostración de un empeoramiento progresivo del estado ácido base en la arteria umbilical en proporción al tiempo transcurrido desde el parto del primer gemelar (86).

Cefálica/no-cefálica: Existe controversia respecto al manejo de los gemelos en presentación cefálica/ no-cefálica, siendo alternativas posibles: operación cesárea electiva para ambos, parto vaginal para el primer gemelo y cesárea para el segundo, y parto vaginal para ambos gemelos, sea con parto en podálica o versión a cefálica (externa o interna) del segundo gemelar.

La revisión sistemática publicada en Cochrane Library mostró que la cesárea electiva no mejora el resultado perinatal y aumenta la tasa de morbilidad febril (87). La decisión de ofrecer parto vaginal a embarazos gemelares en presentación cefálica-no cefálica dependerá del peso de los gemelos; si el peso estimado está entre 1500 y $4000 \mathrm{~g}$ se recomienda parto vaginal si el obstetra está cómodo y tiene experiencia en la atención de partos vaginales en podálica.

El parto vaginal para ambos gemelos luego de versión cefálica externa del segundo gemelo es una alternativa de parto para estos gemelos, ya que con una baja tasa de complicaciones, es posible lograr más del $60 \%$ de parto en cefálica de ambos fetos (84). Sin embargo, consideran más seguro proceder al parto en podálica del segundo gemelar, si el peso de nacimiento y las habilidades del operador, así lo aconsejan (84).

No-cefálica/otra: La presentación no-cefálica/otra es la presentación menos frecuente en gemelos. La presentación del segundo gemelo no es tomada en cuenta ya que el manejo intraparto se rige por la presentación del primer gemelo. En estos casos, se ha descrito un mayor riesgo de entrecruzamiento y mortalidad perinatal al intentar un parto vaginal, por este motivo, se recomienda la operación cesárea electiva en este grupo (88) (Tabla VII).

\section{Tabla VII \\ CUADRO RESUMEN DE RECOMENDACIONES SOBRE MOMENTO DE INTERRUPCIÓN Y MANEJO INTRAPARTO}

\section{MOMENTO DE INTERRUPCIÓN}

1. Embarazos gemelares no complicados interrumpir entre las 37 y 38 semanas.

2. Gemelares con complicación, pero en bienestar fetal, interrumpir a las 36-37 semanas.

3. Gemelares monoamnióticos debieran interrumpirse a las 32 semanas (II-2,3 C).

MANEJO INTRAPARTO

1. Diagnóstico de presentación fetal ultrasonográfico al ingreso a maternidad.

2. Presentación cefálica/cefálica indicación de cesárea similar a gestación única (III, C).

3. Parto vaginal con monitorización intraparto continua, acceso inmediato a operación cesárea, analgésia apropiada y un obstetra con experiencia (B).

4. Si se planea un parto vaginal, estimación de peso fetal ecográfico (A).

5. La versión cefálica externa del segundo gemelo en presentación no cefálica se asocia con una mayor tasa de complicaciones que la extracción en nalgas.

6. En presentación cefálica / no cefálica el parto vaginal con extracción podálica del segundo gemelo debe intentarse solo si la estimación del peso fetal es al menos 1700 gramos y el médico tiene experiencia en atención de partos en podálica.

7. En presentación no-cefálica / otra efectuar cesárea electiva.

8. Gemelares monoamnióticos y gemelos unidos, efectuar cesárea electiva (III, C). 


\section{CONCLUSIONES}

El control prenatal ha demostrado disminuir el riesgo fetal, para lograr un diagnóstico y manejo adecuado de los embarazos gemelares y sus complicaciones, por lo que resulta importante contar con guías clínicas actualizadas. En las aquí presentadas hemos resumido la información actualmente disponible y plantear pautas de diagnóstico y manejo basadas en evidencia.

\section{BIBLIOGRAFÍA}

1. Platt MJ, Marshall A, Pharoah PO: The effects of assisted reproduction on the trends and zygosity of multiple in England and Wales 1974-99. Twin Res 2001;4(6):417-421.

2. Martin JA, Hamilton BE, Sutton PD, et al. Births: final data for 2004. National vital statistics reports. Vol 55. No. 1. Hyattsville, MD: National Center for Health Statistics, 2006.

3. Cunningham FG, Leveno KJ, Bloom SL, Hauth JC, Gilstrap LC, Wenstrom KD. Gestación multifetal. En: Obstetricia de Williams, 22a edición Santa Fe. Editorial McGaw-Hill Interamericana, 2006; 911-948.

4. Lewi L, Deprest J. Fetal Problems in Multiple Pregnancy. En: James DK, Steer PJ, Weiner CP, Gonik B. High Risk Pregnancy: Management Options. Tercera edición. Philadelphia. Elsevier Saunders, 2006; 794846.

5. Creasy RK, Resnik R. Multiple Gestation: Clinical Characteristics and Management. En: Maternal-Fetal Medicine. Quinta edición. Saunders.

6. Woolf SH, Battista RN, Argenson GM, Logan G, Eel W. Canadian Task Force on the Periodic Health Exam. Ottawa: Canada Communication Group, 1994:xxxvii.

7. National Institute for Health and Clinical Excellence. Clinical Guidelines for Fertility assessment and treatment for people with fertility problems 2004. Barlow D, Brimblecombe P, Brown C, Duckitt K, Dunlop J, Hartshorne G. Hallado en: http://www.nice.org.uk/nicemedia/pdf/CG011niceguideline.pdf. Acceso el 10 de Julio de 2008

8. Sepúlveda W, Sebire N, Hughes K, Odibo A, Nicolaides $\mathrm{K}$. The lambda sign at 10-14 weeks of gestation as a predictor of chorionicity in twin pregnancies. UItrasound Obstet Gynecol. 1996; 7: 421-3.

9. The SOGC Consensus Statement: Management of Twin Pregnancies (Part 1). J Soc Obstet Gynaecol Can 2000;22(7):519-29.

10. Sebire NJ, Souka A, Skentou H, Geerts L, Nicolaides $\mathrm{KH}$. Early prediction of severe twin-to-twin transfusion syndrome. Human Reprod. 2000; 15: 2008-10.

11. Casasbuenas A, Wong A, Sepúlveda W. Nuchal translucency thickness in monochorionic multiple pregnancies. Value in predicting pregnancy outcome. J Ultrasound Med. 2008; 27: 363-9.

12. Acosta-Rojas R, Becker J, Munoz-Abellana B, Ruiz C, Carreras E, Gratacos E; for the Catalunya and Balears Monochorionic Network. Twin chorionicity and the risk of adverse perinatal outcome. Int $\mathrm{J}$ Gynaecol Obstet. 2007; 96: 98-102.

13. Sebire NJ, Snijders RJM, Hughes K, Sepúlveda W, Nicolaides $\mathrm{KH}$. The hidden mortality of monochorionic twin pregancies. Br J Obstet Gynecol 1997;104: 12037.

14. Yamasmit W, Chaithongwongwatthana S, Tolosa JE, Limpongsanurak S, Pereira L, Lumbiganon P. Prophylactic oral betamimetics for reducing preterm birth in women with a twin pregnancy. Cochrane Database of Systematic Reviews 2005, Issue 3. Art. No.: CD004733.

15. Martin JA, Hamilton BE, Ventura SJ, et al. Births: Final Data for 2001. Nat Vital Stat Rep 2002;51(2):1-102.

16. Souka AP, Heath V, Flint S, Sevastopoulo I, Nicolaides $\mathrm{KH}$. Cervical length at 23 weeks in twins for predicting spontaneous preterm delivery. Obstet Gynecol 1999;94:450-4.

17. Goldenberg RL, lams JD. The preterm prediction study: risk factors in twin gestations. Am J Obstet Gynecol 1996;175:1047-53.

18. Guzman ER, Walters C, O`Reilly-Green C, et al. Use of cervical ultrasonography in prediction of spontaneous preterm birth in twin gestations. Am J Obstet Gynecol 2000;183(5):1103-7.

19. Goldenberg RL, Mercer BM, Meis PJ, et al. The preterm prediction study: fetal fibronectin testing and spontaneous preterm birth. Obstet Gynecol 1996;87:643-8.

20. Tolino A, Ronsini S, Zullo F, et al. Fetal fibronectin as a screening test for premature delivery in multiple pregnancies. Int J Gynaecol Obstet. 1996;52:3-7.

21. Olivera T, de Souza E, Mariani-Neto C, Camano L. Fetal fibronectin as a predictor of preterm delivery in twin gestations. Int J Gynaecol Obstet 1998;62:1359.

22. Crowther CA. Hospitalization and bed rest for multiple pregnancy. Cochrane Database Syst Rev 2001;(1): CD000110.

23. MacLennan AH, Green RC, O'Shea R, Brookes C, Morris D. Routine hospital admission in twin pregnancy between 26 and 30 weeks gestation. Lancet 1990;335(8684):267-9.

24. Andrews WW, Leveno KJ, Sherman ML, et al. Elective hospitalization in the management of twin pregnancies. Obstet Gynecol 1991;77:826-31.

25. Berghella V, Odibo AO, To MS, Rust OA, Althusius SM. Cerclage for short cervix on ultrasonography: meta-analysis of trials using individual patient-level data. Obstet Gynecol. 2005;106:181-9.

26. Yamasmit W, Chaithongwongwatthana S, Tolosa JE, Limpongsanurak S, Pereira L, Lumbiganon P. Prophylactic oral betamimetics for reducing preterm birth in women with a twin pregnancy. Cochrane Database of Systematic Reviews 2005, Issue 3. Art. No.: CD004733.

27. Rouse DJ, Caritis SN, Peaceman AM. ATrial of 17 Alpha-Hydroxiprogesterone Caproate to Prevent Prematurity in Twins. N Engl J Med 2007;357:454-61.

28. Ananth CV, Vintzileos AM, Shen-Schwarz S, et al: Standards of birth weight in twin gestations stratified by placental chorionicity. Obstet Gynecol 1998; 91:917-924. 
29. Hamilton EF, Platt RW, Morin L, et al: How small is too small in a twin pregnancy?. Am J Obstet Gynecol 1998; 179:682-685.

30. Sebire N, Snijders R, Hughes K, et al: The hidden mortality of monochorionic twin pregnancies. BJOG 1997; 104:1203-1207.

31. Gratacós E, Lewi L, Carreras E, Becker J, Higueras T, Deprest $\mathrm{J}$ et al. Incidence and characteristic of umbilical intermittent absent and/or reversed end-diastolic flow in complicated and uncomplicated monochorionic twin pregnancies. Ultrasound Obstet Gynecol. 2004; 23: 456-460.

32. Hill LM, Guzick D, Cheveney P, et al: The assessment of twin growth discordancy. Obstet Gynecol 1994; 84:501-504.

33. Sebire NJ, D'Ercole C, Hughes K, et al: Dichorionic twins discordant for intrauterine growth retardation. Arch Dis Child 1997; 77:F235-F236.

34. Whitworth NS, Magann EF, Morrison JC: Evaluation of fetal lung maturity in diamniotic twins. Am J Obstet Gynecol 1999; 180(6 Pt 1):1438-1441.

35. Nakai $\mathrm{Y}$, Ishoko $\mathrm{O}$, Nishio J, et al: Cyclic changes in the umbilical arterial flow in monochorionic diamniotic twin pregnancy. Eur J Obstet Gynecol Reprod Biol 2002; 101:135-138.

36. Gratacós E, Antolin E, Lewi L, Martínez JM, Hernandez-Andrade E, Acosta-Rojas R. Monochorionic twins with selective intrauterine growth restriction and intermittent absent or reversed end-diastolic flow (Type III): feasibility and perinatal outcome of fetoscopic placental laser coagulation. Ultrasound Obstet Gynecol 2008;31(6):669-75.

37. Norton ME, D'Alton ME, Bianchi DW: Molecular zygosity studies aid in the management of discordant multiple gestations. J Perinat 1997;17(3):202-7.

38. Joern H, Klein B, Schmid-Schoenbein H, Rath W: Antenatal visualization of vascular anastomoses in monochorionic twins using colour Doppler sonography: The protective function of these anastomoses and the phenomenon of interference beating. Ultrasound Obstet Gynecol 1999;14:422-5.

39. Kiely JL: The epidemiology of perinatal mortality in multiple births. Bull NY Acad Med 1990; 66:618-637

40. Tummers $P$, De Sutter P, Dhont M: Risk of spontaneous abortion in singleton and twin pregnancies after IVF/ICSI. Hum Reprod 2003;18(8):1720-1723.

41. Sebire NJ, Thornton S, Hughes K, et al: The prevalence and consequences of missed abortion in twin pregnancies at 10 to 14 weeks of gestation. BJOG 1997; 104:847-848.

42. ACOG Technical Bulletin N 176 January 1993: Diagnosis and management of fetal death. Int $\mathrm{J}$ Gynecol Obstet 1993; 42: 291-299.

43. Johnson CD, Zhang J: Survival of other fetuses after a fetal death in twin or triplet pregnancies. Obstet Gynecol 2002; 99:698-703.

44. Bajoria R, Wee LY, Anwar S, Ward S: Outcome of twin pregnancies complicated by single intrauterine death in relation to vascular anatomy of the monochorionic placenta. Hum Reprod 1999; 14(8):2124-2130.

45. Nicolini U, Poblete A: Single intrauterine death in monochorionic twin pregnancies. Ultrasound Obstet Gynecol 1999; 14:297-301.
46. Woo HHN, Sin SY, Tang LCH: Single fetal death in twin pregnancies: Review of the maternal and neonatal outcomes and management. Hong Kong Medical Journal 2001; 6:293-300.

47. Quarello E, Stirnemann J, Nassar M, Nasr B, Bernard $\mathrm{JP}$, Leleu-Huard $\mathrm{F}$, et al. Outcome of anaemic monochorionic single survivors following early intrauterine rescue transfusion in cases of feto-fetal transfusion syndrome. Br J Obstet Gynecol. 2008; 115: 595-601.

48. Edwards M, Ellings J, Newman R, Menard M: Predictive value of antepartum ultrasound examination for anomalies in twin gestations. Ultrasound Obstet Gynecol 1995; 6:43-49.

49. Bryan E, Little J, Burn J: Congenital anomalies in twins. Baillieres Clin Obstet Gynecol 1987; 1:697-721.

50. Lewi L, Deprest J. Fetal Problems in Multiple Pregnancy. En: James DK, Steer PJ, Weiner CP, Gonik B. High Risk Pregnancy: Management Options. Tercera edición. Philadelphia. Elsevier Saunders, 2006; 829.

51. Wald NJ, Rish S, Hackshaw AK: Combining nuchal translucency and serum markers in prenatal screening for Down syndrome in twin pregnancies. Prenat Diagn 2003;23(7):588- 592.

52. Spencer K, Nicolaides KH: Screening for trisomy 21 in twins using first trimester ultrasound and maternal serum biochemistry in a one-stop clinic: A review of three years experience. BJOG 2003; 110(3): 276280.

53. Hanson J: Incidence of conjoining twinning. Lancet 1975; ii:1257.

54. Barth R, Filly R, Goldberg J, et al: Conjoined twins: Prenatal diagnosis and assessment of associated malformations. Radiology 1990;177:201-207.

55. Huber A, Hecher K. How can we diagnose and manage twin-twin transfusion syndrome? Best Prac Clin Obstet Gynecol. 2004; 18: 543-56.

56. Quintero RA, Morales WJ, Allen $\mathrm{MH}$, et al. Staging of twin-to-twin transfussion syndrome. J Perinatol.1999;19:550-555.

57. Denbow ML, Battin MR, Cowan F, et al: Neonatal cranial ultrasound findings in preterm twins complicated by severe fetofetal transfusion syndrome. Am J Obstet Gynecol 1998; 178:479-483.

58. Lopriore E, Nagel HT, Vandenbussche FP, Walther FJ: Long-term neurodevelopmental outcome in twinto-twin transfusion syndrome. Am J Obstet Gynecol 2003; 189(5):1314- 1319.

59. Mari G, Roberts A, Detti L, Kovanci E, Stefos T, Bahado-Singh $\mathrm{RO}$ et al. Perinatal morbidity and mortality rates in severe twin-to-twin transfusion syndrome: Results of the International Amnioreduction Registry. Am J Obstet Gynecol. 2001; 185: 708-15.

60. Senat MV, Deprest J, Boulvain M, et al: A randomized trial of endoscopic laser surgery versus serial amnioreduction for severe twin-to-twin transfusion syndrome at midgestation. N Engl J Med 2004; 35(2):136-144.

61. Yamamoto $M$, Ville $Y$. Laser treatment in twin-to-twin transfusion syndrome. Semin Fetal Neonat Med. 2007; 12:450-7.

62. Roberts D, Neilson JP, Kilby M, Gates S. Interventions for the treatment of twin-twin transfusion syndrome. Cochrane Database of Systematic Reviews 2008, Issue 1. Art. No.: CD002073. 
63. Sebire NJ, Souka A, Skentou $H$, et al: First trimester diagnosis of monoamniotic twin pregnancies. Ultrasound Obstet Gynecol 2000; 16:223-225.

64. Arabin B, Laurini RN, Van Eyck J: Early prenatal diagnosis of cord entanglement in monoamniotic multiple pregnancies. Ultrasound Obstet Gynecol 1999; 13:181-186.

65. Timmons JD, DeAlvarez RR: Monoamniotic twin pregnancy. Am J Obstet Gynecol 1963; 86:875-881.

66. Rodis J, Mcllveen P, Egan J, et al: Monoamniotic twins: improved perinatal survival with accurate prenatal diagnosis and antenatal fetal surveillance. Am J Obstet Gynecol 1997; 177:1046-1049.

67. Allen VM, Windrim R, Barrett J, Ohlsson A: Management of monoamniotic twin pregnancies: A case series and systemic review of the literature. BJOG 2001; 108:931- 936.

68. Abuhamad AZ, Mari G, Copel JA, et al: Umbilical artery flow velocity waveforms in monoamniotic twins with cord entanglement. Obstet Gynecol 1995; 86:674677.

69. Su LL: Monoamniotic twins: Diagnosis and management. Acta Obstet Gynecol Scand 2002; 81:9951000.

70. Roque H, Gillen-Goldstein J, Funai E, Young BK, Lockwood CJ. Perinatal outcomes in monoamniotic gestations. J Matern Fetal Neonatal Med 2003; 13(6); 414-21.

71. Schwarzler P, Ville $Y$, Moscosco G, et al: Diagnosis of twin reversed arterial perfusion sequence in the first trimester by transvaginal color Doppler ultrasound. UItrasound Obstet Gynecol 1999; 13(2):143-146.

72. Sebire N, Wong A, Sepúlveda W. Minimally invasive management of twin reversed arterial perfusion sequence (TRAP). Fetal Mater Med Rev. 2006; 17: 122.

73. Dashe JS, Fernandez CO, Twickler DM: Utility of Doppler velocimetry in predicting outcome in twin reversed-arterial perfusion sequence. Am J Obstet Gynecol 2001; 185(1):135-139.

74. Wong A, Sepúlveda W. Acardiac anomaly: current issues in prenatal assessment and treatment. Prenat Diagn. 2005; 25: 796-806.

75. Holzgreve W, Tercanli S, Krings W, Schuierer G: A simpler technique for umbilical-cord blockade of an acardiac twin. N Engl J Med 1994; 331(1):56-57.
76. Quintero RA, Reich $\mathrm{H}$, Puder KS, et al: Brief report: Umbilical cord ligation of an acardiac twin by fetoscopy at 19 weeks of gestation. N Engl J Med 1994; 330(7):469-471.

77. Deprest J, Audibert F, Van Schoubroeck D, et al: Bipolar cord coagulation of the umbilical cord in complicated monochorionic twin pregnancy. Am J Obstet Gynecol 2000; 182:340-345.

78. Ville Y, Hyett JA, Vandenbussche FP, Nicolaides KH: Endoscopic laser coagulation of umbilical cord vessels in twin reversed arterial perfusion sequence. UItrasound Obstet Gynecol 1994; 4(5):396-398.

79. Rodeck C, Deans A, Jauniaux E: Thermocoagulation for the early treatment of pregnancy with an acardiac twin. N Engl J Med 1998; 339:1293-1294.

80. Luke B. Reducing fetal deaths in multiple births: optimal birthweights and gestational ages for infants of twin and triplet births. Acta Genet Med Gemellol (Roma). 1996;45:333-348.

81. Chang EY. Timing of Delivery in Multiple Gestation. Clin Obtet Gynecol 2004;47(1):237-247.

82. Suzuki S, Otsubo Y, Sawa R, Yoneyama Y, Araki T. Clinical trial of inductionof labor versus expectant management in twin pregnancy. Gynecol Obstet Invest 2000;49:24-7.

83. Dodd JM, Crowther CA. Elective delivery of women with a twin pregnancy from 37 weeks' gestation. Cochrane Database of SystematicReviews 2003, Issue 1. Art. No.: CD003582.

84. Robinson S, Chauhan S. Intrapartum Management of Twins. Clin Obstet Gynecol 2004;47(1):248-262.

85. Smith GCS, Pell JP, Dobbie R. Birth order, gestational age, and risk of delivery related perinatal death in twins: retrospective cohort study. BMJ 2002;325:10046.

86. Leung TY, Tam WH, Leung TN, Lok IH, Lau TK. Effect of twin-to-twin delivery interval on umbilical cord blood gas in the second twins. BJOG. 2002;109: 63-7.

87. Crowther CA. Caesarean delivery for the second twin. Cochrane Database of Systematic Reviews 1996, Issue 1. Art. No.: CD000047.

88. American College of Obstetricians and Gynecologists. Special problems of multiple gestation. ACOG educational bulletin No.253. Washington DC: ACOG, 1998. 